\title{
VALIDACIÓN DE ESCALA SUBJETIVA DE CARGA MENTAL DE TRABAJO EN FUNCIONARIOS/AS UNIVERSITARIOS ${ }^{1}$
}

\section{VALIDATION OF SUBJECTIVE MENTAL WORKLOAD SCALE IN ACADEMICS STAFF}

\author{
Paula Ceballos Vásquez * \\ Tatiana Paravic Klijn ${ }^{* *}$ \\ Mónica Burgos Moreno ${ }^{* * x}$ \\ OMAR BARRIGA ${ }^{* * *}$
}

\begin{abstract}
RESUMEN
Objetivo: Evaluar las características psicométricas de la Escala Subjetiva de Carga Mental de Trabajo (ESCAM). Material y método: La población de este estudio estuvo constituida por 56 personas de ambos sexos, obtenidos a través de un censo a los y las funcionarios/as de dos facultades de una universidad chilena, quienes respondieron la Escala Subjetiva de Carga Mental de Trabajo y cuestionario de Evaluación de Riesgos Psicosociales. La fiabilidad fue comprobada con el coeficiente de alfa de Cronbach; para la validez de contenido, la escala fue revisada por expertos ad hoc; la validez de constructo fue evaluada por análisis factorial con rotación Varimax, y la validez de criterio se midió al correlacionar los resultados de la escala con el instrumento validado SUSESO-ISTAS 21. Resultados: Se obtuvo una adecuada confiabilidad $(\alpha=0.79)$, además una validez de contenido por análisis de expertos y prueba piloto; validez de criterio por análisis factorial; y validez de constructo por correlación de Pearson, las que confirmaron la estructura multidimensional de escala. Conclusiones: ESCAM es un instrumento fiable y válido para evaluar la percepción de Carga Mental de Trabajo.
\end{abstract}

Palabras clave: Validez, reproducibilidad de resultados, salud ocupacional, enfermería del trabajo, carga mental de trabajo.

\footnotetext{
ABSTRACT

Objective: Evaluate the psychometric characteristics of the Subjective Mental Workload Scale (SMWS). Materials and methods: the sample of this study consisted of 56 persons of both genders, obtained through a census of the staff of two faculties of a Chilean university, who answered the Subjective Mental Workload Scale and the psychosocial risks evaluation questionnaire. The reliability was verified by Cronbach alpha coefficient, for the validity of content, the scale was checked by ad hoc experts. Constructo validity was evaluated by factorial analysis with Varimax rotation, and the criterion validity was measured when the results of the scale were correlated with the validated instrument SUSESO-ISTAS 21. Results: the multidimensional structure of the

${ }^{1}$ Trabajo derivado de Tesis "Percepción de riesgos psicosociales y carga mental de trabajo del equipo de salud en unidades de paciente crítico", Programa Doctorado en Enfermería, Universidad de Concepción con aporte de Proyecto MESESUP UBB0607.

*Enfermera. Docente Departamento de Enfermería. Universidad Católica del Maule. Talca, Chile. E-mail: pauceballos@ udec.cl.

${ }^{* *}$ Enfermera. Docente Departamento de Enfermería. Facultad de Medicina, Universidad de Concepción. Concepción, Chile. E-mail: tparavic@udec.cl.

${ }^{* * *}$ Enfermera. Docente Departamento de Enfermería. Facultad de Medicina, Universidad de Concepción. Concepción, Chile.E-mail: moburgos@udec.cl.

${ }^{* * * *}$ Sociólogo. Docente Departamento Socialogía y Antropología, Universidad de Concepción. Concepción, Chile. E-mail: obarriga@udec.cl.
} 
scale was confirmed, with a suitable reliability $(\alpha=0.79)$, the criterion validity by factorial analysis, and the constructo validity by Pearson correlation. Conclusion: SMWS is indicated as a reliable and valid instrument to evaluate the Mental Workload Perception.

Key words: Validity, reproducibility of results, occupational health, occupational health nursing, mental workload.

Fecha recepción: 04/09/13. Fecha aceptación: 25/04/14.

\section{INTRODUCCIÓN}

La sociedad actual ha llevado a la humanidad a una espiral de actividad-trabajo que rebasa la carga física de trabajo, y que genera sobrecarga mental que conduce al estrés. Esto conlleva a que la evaluación de la Carga Mental de Trabajo sea un aspecto central para la investigación y el desarrollo de sistemas preventivos en el ámbito laboral, que permitan obtener niveles más altos de confort, satisfacción, eficacia y seguridad laboral (1-4).

La Carga Mental constituye un importante factor dentro de los riesgos psicosociales a nivel laboral. Sin embargo, aún no existe consenso en su definición, especialmente la de tipo operacional $(1,5-6)$. En esta investigación se entenderá como Carga Mental de Trabajo (CMT) a la brecha existente entre la capacidad de la persona para realizar una tarea y los requerimientos de ésta, como a los recursos necesarios para desarrollarla. Además se postula que es una variable multidimensional, ya que está compuesta por diversos factores o dimensiones (7-8), por lo que su medición debe incorporarlas.

Existen variados tipos de medición para la CMT, entre ellas se encuentra: medidas de ejecución, medidas fisiológicas y medidas subjetivas $(1,8)$. Aunque se postulan las ventajas y desventajas de todas ellas, investigadores/as indican que las medidas subjetivas serían el procedimiento más adecuado para la evaluación de $\operatorname{CMT}(1,9)$. Éstas permiten conocer la percepción del trabajador/a respecto a la Carga Mental que le supone la realización de la tarea. Además, los instrumentos que miden subjetivamente la carga mental de trabajo son los utilizados con más frecuencia en contextos aplicados por su elevada aceptación por los trabajadores, buena validez y fiabilidad $(7,9)$.

Así, el 2009 nace la Escala Subjetiva de Carga Mental de Trabajo (ESCAM). Sus autoras indican que la Carga Mental debe abordarse desde una perspectiva integral incorporando, en una medida, elementos del enfoque exigencias-recursos, como elementos del enfoque exigencias de la tarea $(1,6$, 10-12). Esta nueva escala emerge con el objetivo de crear un instrumento que superará las limitaciones de otras escalas existentes (6). Sus creadoras postulan que empíricamente las dimensiones de Carga Mental de Trabajo son: Demandas cognitivas y complejidad de la tarea, Característica de la tarea, Organización temporal, Ritmo de trabajo y Consecuencias para la salud $(6,13)$.

La presente investigación tiene por objetivo evaluar las características psicométricas de la Escala Subjetiva de Carga Mental de Trabajo (ESCAM) en población chilena, con la finalidad de que investigadores/as en la temática, dispongan de un instrumento que les permita medir Carga Mental de Trabajo. Especialmente para los y las enfermeros/as dedicados a la Enfermería Ocupacional, ya que podrán contar con un instrumento validado para el contexto nacional y así cubrir los vacíos de conocimiento en relación a la Carga mental, además de proponer estrategias para mejorar los contextos de trabajos desde la perspectiva de Enfermería. 


\section{MATERIAL Y MÉTODO}

Para esta validación se censó a los y las trabajadores/as de dos facultades de una institución universitaria chilena, donde participó el $74,5 \%$ del total de trabajadores/as lo que equivale a 56 personas de ambos sexos, quienes respondieron la Escala Subjetiva de Carga Mental de Trabajo (ESCAM) y cuestionario de evaluación de riesgos psicosociales (SUSESO ISTAS-21), previa firma de consentimiento libre e informado. En relación a las características del grupo estudiado, se puede indicar que el $80,4 \%$, fueron mujeres. Sus edades fluctuaron entre 24 y 62 años, con una media de 40,2 años. Referente al estado civil, el 51,8\% reportaron estar casados/as y el 30,3\% solteros/as. En relación a la profesión de los y las participantes, el $37 \%$ fueron enfermeras/os, el 16,1\% kinesiólogos/as, el 12,5\% psicólogos/as, el 14,3\% administrativos/as, el 3,6\% correspondieron a Obstetras, $\mathrm{y}$ en porcentajes iguales $(5,4 \%)$ a médicos, a nutricionistas y a personal auxiliar. En relación a su situación laboral, el 75\% de los funcionarios se encontraban con contrato indefinido y el $100 \%$ de los trabajadores se desempeñan en jornada diurna.

Este estudio fue aprobado por el Comité de Bioética de la Facultad de Medicina de la Universidad de Concepción, atendiendo al DIFM 010/2013. Además se solicitó autorización a las autoridades de las Facultades participantes.

Para realizar la evaluación psicométrica de ESCAM, se evaluó la confiabilidad y la validez (14). La confiabilidad es un criterio importante para evaluar su calidad y la idoneidad de los reactivos (15). Se refiere al grado en que su aplicación repetida al mismo sujeto $\mathrm{u}$ objeto produce resultados iguales (16). Para ello, se calculó el alfa de Cronbach, que mide la consistencia interna de la escala (17). Posteriormente se estimó la validez que, según especialistas, "denota el grado en que el instrumento mide lo que se supone debe medir" $(15,18)$. La validez de contenido de ESCAM fue realizada a través de expertos del área de la psicología laboral, ergonomía y enfermería laboral, y una prueba piloto con enfermeras/os asistenciales, quienes no fueron incluidas/os en este estudio. Luego, para estimar la validez de constructo, se realizó un análisis factorial exploratorio de componente principales con rotación ortogonal (Varimax). Finalmente se evaluó la validez de criterio concurrente, por medio de análisis de correlación, entre las dimensiones de ESCAM y SUSESO ISTAS-21 (Dimensión exigencias psicológicas). Se indica que la organización, el procesamiento y análisis estadístico de los datos se realizó con el software SPSS versión 15.0 para Windows XP.

\section{Instrumentos}

ESCAM fue creada por docentes del Departamento de Psicología de la Universidad de la Laguna (Tenerife, España). Está compuesta por 5 dimensiones que entregan información multidimensional sobre-Demandas cognitivas y complejidad de la tarea, se relaciona al esfuerzo cognitivo que supone el desempeño del puesto de trabajo. -Consecuencias para la salud, hace referencia al agotamiento que el desempeño de la tarea produce en el trabajador/a. -Características de la tarea, describe las interrupciones y distracciones que se generan en el puesto de trabajo. -Organización temporal, recoge valoraciones sobre la adecuación del tiempo del que dispone el trabajador/a para ejecutar su labor, y -Ritmo de trabajo, se refiere a la posibilidad que tiene el trabajador/a de organizar el tiempo en el desempeño de la tarea, así como el efecto de un error en los resultados del trabajo. Consta de 20 ítemes. Las respuestas son de tipo Likert, con un puntaje mínimo de 1 y un máximo de 5. Se obtiene puntaje total y por cada dimensión, donde las puntuaciones bajas indican menor percepción de carga mental de trabajo y las altas corresponden a mayor percepción de ésta $(6,13)$. 
El cuestionario SUSESO ISTAS-21 versión breve, dirigido a la evaluación de factores psicosociales, es una escala tipo Likert donde cada pregunta tiene 5 opciones de respuesta con una puntuación de 0 a 4 puntos. Cuenta con 20 ítemes agrupados en cinco dimensiones: -Exigencias psicológicas de tipo cognitivas, cuantitativas, emocionales y sensoriales. -Trabajo activo y desarrollo de habilidades, que incluye influencia, autonomía, sentido del trabajo y oportunidades de desarrollo. -Apoyo social en la empresa y calidad de liderazgo, referido a las características del liderazgo ejercido y apoyo socio-instrumental por parte de superiores o compañeros. -Compensaciones, engloba estima percibida, inseguridad respecto a las condiciones laborales y al trabajo ejecutado; y -Doble presencia, referida a la cantidad de trabajo doméstico y/o familiar que depende del trabajador/a, y a la preocupación que las tareas del trabajo doméstico y/o familiar le producen durante el trabajo. Este cuestionario entrega el nivel de riesgo percibido por las/os trabajadoras/ es, que se clasifica en bajo, medio y alto. Dicho instrumento se encuentra adaptado, validado y estandarizado para la población trabajadora chilena y está disponible para su utilización (19).

\section{RESULTADOS}

\section{Análisis descriptivo de ESCAM}

Al realizar el análisis univariado, se observa tendencia cercana a la curva normal, lo que se sustenta con el resultado del test de Kolmogorov-Smirnov $(K-S)$, el cual resulta no significativo para las 5 dimensiones de ESCAM, por lo que no hay elementos suficientes para aceptar la hipótesis del test $K-S$, lo que apoyaría el supuesto de normalidad de los datos (Tabla 1).

Tabla 1. Medidas estadísticas descriptivas de ESCAM.

\begin{tabular}{lcccccc}
\cline { 2 - 6 } & $\begin{array}{c}\text { Dimensión 1 } \\
\text { Demandas } \\
\text { cognitivasy } \\
\text { complejidad } \\
\text { de la tarea }\end{array}$ & $\begin{array}{c}\text { Dimensión 2 } \\
\text { Características } \\
\text { de la tarea }\end{array}$ & $\begin{array}{c}\text { Dimensión 3 } \\
\text { Organización } \\
\text { temporal } \\
\text { del trabajo }\end{array}$ & $\begin{array}{c}\text { Dimensión 4 } \\
\text { Ritmo de } \\
\text { trabajo }\end{array}$ & $\begin{array}{c}\text { Dimensión 5 } \\
\text { Consecuencias } \\
\text { para la salud }\end{array}$ & $\begin{array}{c}\text { Promedio } \\
\text { total Carga } \\
\text { Mental de } \\
\text { Trabajo }\end{array}$ \\
\hline Media & 3,68 & 3,56 & 2,9 & 2,36 & 3,26 & 3,15 \\
Desviación típica & 0,468 & 0,598 & 0,715 & 0,824 & 0,898 & 0,45 \\
Asimetría & $-0,064$ & $-0,41$ & 0,325 & 0,426 & $-0,445$ & $-0,176$ \\
K-S & 0,681 & 1,089 & 0,996 & 0,832 & 1,185 & 0,446 \\
Sig. Asintót (bilateral) & 0,743 & 0,187 & 0,274 & 0,493 & 0,121 & 0,989 \\
\hline
\end{tabular}

$n=54$

\section{Confiabilidad de ESCAM}

En primer lugar, se realizó una depuración estadística de los datos comprobándose el supuesto de ausencia de valores atípicos multivariados, razón por lo que la muestra quedó configurada en 54 personas. La consistencia interna de ESCAM fue de $\alpha=0,79$, lo cual indica que el 79\% de la variabilidad de las puntuaciones obtenidas representan diferencias verdaderas entre las personas y el $21 \%$ reflejan puntuaciones azarosas. Esto permite aseverar que los reactivos son homogéneos y que ESCAM mide en forma consistente el constructo para la que fue elaborada. La Tabla 2 presenta las mediciones del $\alpha$ para cada 
Validación de Escala Subjetiva de Carga Mental de Trabajo en funcionarios/as universitarios / P. Ceballos, T. Paravic, M. Burgos, O. Barriga

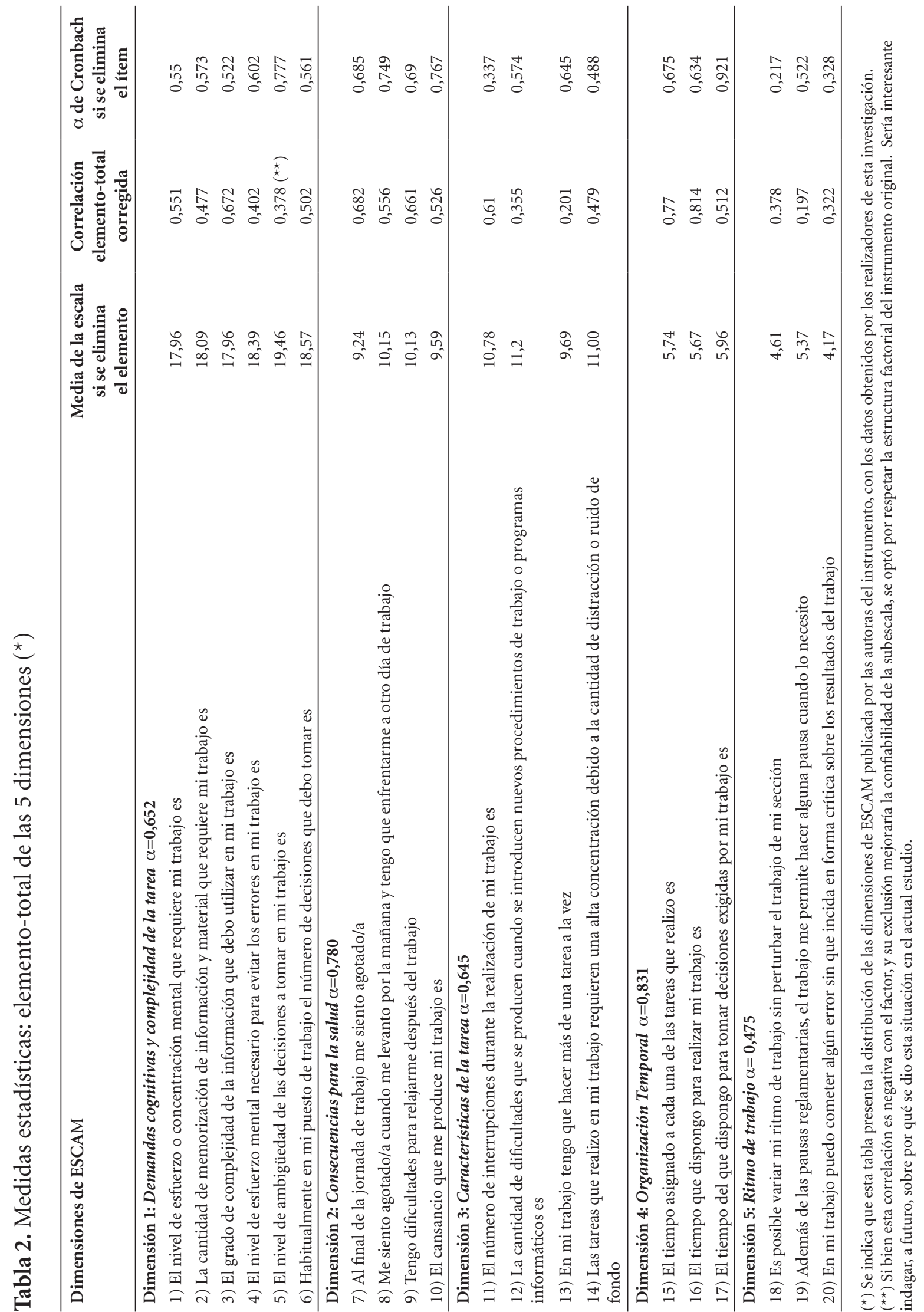


CIENCIA Y ENFERMERIA XX (2), 2014

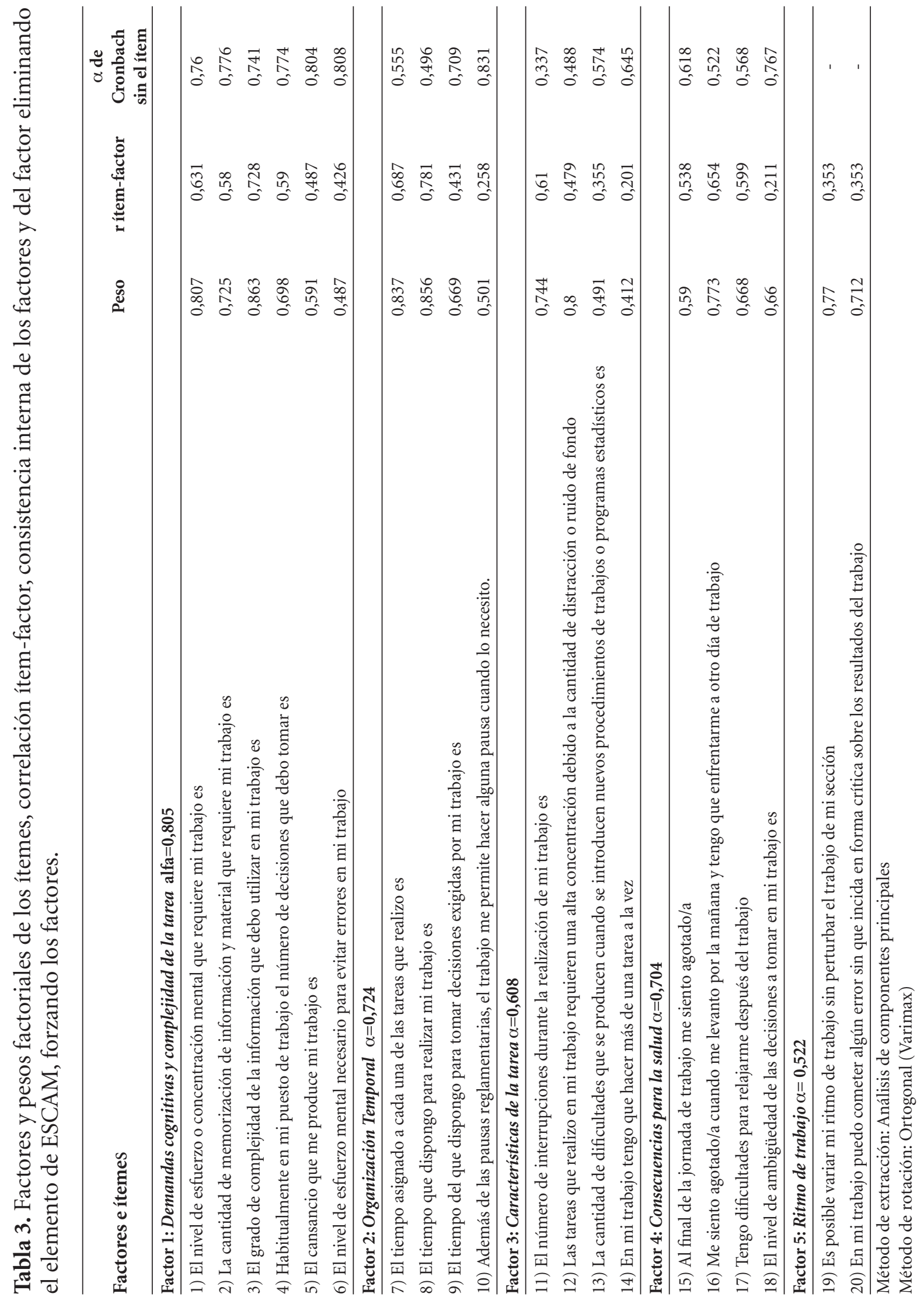


una de las subescalas, donde cuatro de las dimensiones tienen alfa aceptables, sin embargo la quinta dimensión resulta con un $\alpha$ bajo.

\section{Validez de contenido de ESCAM}

Con el fin de determinar el grado de comprensión de los instrumentos a utilizar, se realizó validación por medio de expertos del área de la psicología laboral, ergonomía y enfermería laboral. Y luego, una prueba piloto con un turno de enfermeras/os asistenciales $(n=14)$, quienes eligieron la aplicación de los instrumentos por medio de entrevista. El tiempo de aplicación fue de aproximadamente 15 a 20 min. Respecto de la comprensión de las escalas y cuestionarios, no se encontraron dificultades.

\section{Validez de constructo de ESCAM}

La Tabla 3 exhibe los resultados del análisis factorial de componentes principales. Previo a éste, se calculó el coeficiente de adecuación muestral KMO de 0,647 y un valor de prueba en la esfericidad de Bartlett de $c^{2}(190)=436,257 \mathrm{p} \leq .000$. Ambas pruebas indican que se puede llevar a cabo el análisis factorial (20). Con el fin de examinar los datos con la estructura presentada por las autoras creadoras de ESCAM, se analizan los 20 ítemes del instrumento y se fuerza la exploración de éstos con 5 factores y en ellos se incluyeron los ítemes con saturaciones sobre 0,4. Así, el primer factor, Demandas cognitivas y complejidad de la información, se forma con 6 ítemes y explica el 24,24\% de la varian$\mathrm{za}$, con una adecuada consistencia interna. El segundo factor consta de 4 ítemes, y se denomina Organización temporal, éste revela el $13,23 \%$ de la varianza. Se indica que al eliminar el ítem 10, la confiabilidad de este factor aumenta. Luego el tercer factor se estructuró con 4 ítemes, denominado Características de la tarea, el cual explica el 9,13\% de la varianza, con un alfa de 0,608 , el que aumenta al eliminar el ítem 14. El cuarto factor, denominado Consecuencias para la salud, manifiesta el $8,17 \%$ de la varianza con un alfa sobre 0,7 , el que aumenta al prescindir del ítem 18. Finalmente el quinto factor está conformado por 2 ítemes, se denominó Ritmo de trabajo, el que explicaría el 6,45\% de la varianza, con una consistencia interna baja. El coeficiente del alfa de Cronbach total de esta escala es de 0,793 y explica el $61,25 \%$ de la varianza total.

\section{Validez de criterio concurrente de ESCAM}

Ésta se llevó a cabo correlacionando ESCAM con el instrumento SUSESO-ISTAS 21, específicamente con la dimensión exigencias psicológicas. En la Tabla 4 se observa, que la correlación es relativamente alta, con un $\mathrm{p} \leq .000$ (Tabla 4).

Tabla 4. Correlación entre percepción de Carga Mental de Trabajo y evaluación de riesgos psicosociales y su dimensión Exigencias Psicológicas.

\begin{tabular}{lcc}
\hline & $\begin{array}{c}\text { Correlación con ESCAM } \\
\text { con AF forzado }\end{array}$ & $n$ \\
\hline $\begin{array}{l}\text { SUSESO ISTAS -21 } \\
\text { EXIGENCIAS PSICOLÓGICAS }\end{array}$ & DIMENSIÓN & $0,735^{(* *)}$ \\
\hline${ }^{(*)}$ La correlación es significativa a nivel de 0,01 (bilateral) & 54
\end{tabular}




\section{DISCUSIÓN}

Los hallazgos de validez y fiabilidad de ESCAM, en una muestra de trabajadores/as chilenos, indican que dicha escala funciona razonablemente bien. Se postula que la utilización de instrumentos validados en las investigaciones de enfermería habilita la divulgación de los hallazgos, potencia los resultados obtenidos, permite la comparación con otras investigaciones y refuerzan las líneas investigativas. Adicionalmente, el uso de ESCAM proporcionaría a enfermeros y enfermeras de salud laboral la posibilidad de realizar diagnósticos en los lugares de desempeño, los que apoyarían la implementación de estrategias de promoción y prevención en relación a este tipo de riesgos psicosociales, con el fin de impulsar ambientes de trabajo más saludables.

En relación a la fiabilidad de ESCAM, se demuestra su homogeneidad, al obtener un adecuado coeficiente de alfa de Cronbach $(0,79)$ en la escala global, el cual es más alto que el reportado por autores españoles $(0,75)$ creadores de la escala, y un poco más bajo que el encontrado en un estudio realizado en docentes universitarios ecuatorianos $(0,858)$ (21).

Por otra parte, al realizar el análisis por factor, en las Demandas cognitivas y complejidad de la tarea el $\alpha$ obtenido es relativamente bajo (17), pero al eliminar el ítem 5 , aumenta sobre 0,7 . Además, se visualiza que la tercera dimensión presenta un $\alpha$ menor a 0,7 , el cual no mejora al eliminar ningún ítem de la dimensión. Finalmente, se observa que el $\alpha$, en la dimensión Ritmo de trabajo es bajo, al igual que el reportado por sus creadoras, aunque en esta muestra adquiere un valor más alto (6), y aumenta al eliminar uno de los ítemes, pero esto hace que quede la dimensión con menos de tres ítemes, por lo que no permitiría su análisis (22). Sumado a ello, el alfa global no mejora en gran medida su valor al eliminar estos ítemes. De esta ma- nera se confirma lo planteado por las creadoras de ESCAM, en relación a que la tercera y quinta dimensión no serían centrales para la medición subjetiva de la carga mental de trabajo, o que quizá es necesario incorporar otros ítemes en estas dimensiones (6).

Para la validez de constructo se realizó análisis factorial y rotación Varimax. El análisis se realizó forzando los datos a cinco factores, estructura presentada por las creadoras de ESCAM. En general, los ítemes se ordenan de manera muy similar a la publicada, pero el orden de los factores se altera y algunos ítemes se agregan en otras dimensiones. La primera dimensión se mantiene con seis ítemes, pero el ítem, el nivel de ambigüedad de las decisiones a tomar en mi trabajo, se traslada al cuarto factor, y la consistencia interna del factor mejora en relación a la publicada por un estudio español (6). El factor Organización temporal, en esta investigación pasa a ser segundo y la rotación incorpora el ítem además de las pausas reglamentarias, el trabajo me permite hacer alguna pausa cuando lo necesito, el cual no mejora la confiabilidad de la dimensión por lo que se sugiere descartar dicho ítem. El tercer factor Características de la tarea se mantiene con los mismos ítemes presentados por sus creadoras, con una mejor fiabilidad. El cuarto factor que entrega este análisis, Consecuencias para la salud, rota uno de sus ítemes con el primer factor, el ítem el cansancio que produce mi trabajo, y aporta en la mejora de la confiabilidad del factor, según lo publicado por las autoras de ESCAM (6). Finalmente, el quinto factor, Ritmo de trabajo, es el mismo que presentan las autoras de ESCAM, pero en él sólo pesan dos ítemes, lo que complicaría su análisis por criterio teórico el cual ya fue comentado con anterioridad; sería importante investigar el aporte empírico y teórico de esta dimensión para la medición subjetiva de CMT. Como se puede observar el ajuste de los datos no es exacto al publicado por las creadoras del instrumento, pero los cambios son mínimos y con una mejor fiabilidad de todas las subes- 
calas y la escala global.

Se indica que se efectuaron otros análisis factoriales en este estudio, con el fin de visualizar como se estructuraban los ítemes sin forzar las dimensiones. De éstos resultaron diversas estructuras factoriales, las cuales presentaron mayores desventajas que la presentada, sin embargo los datos obtenidos de éstos son interesantes aunque irrelevantes para esta validación.

Con el fin de medir la validez de criterio (concurrente), se correlacionó ESCAM con SUSESO-ISTAS 21. La correlación se realizó con la estructura factorial y la dimensión Exigencias psicológicas de la escala criterio. Dicha dimensión mide las exigencias psicológicas cuantitativas, exigencias psicológicas cognitivas y exigencias psicológicas emocionales (19), aspectos que se relacionan directamente con la carga mental a nivel laboral. Se obtuvo una correlación positiva entre ESCAM y el instrumento criterio, lo que apoya la validez de la escala, ya que el coeficiente de validez es alto.

Dentro de las limitaciones presentes en este estudio, se pueden mencionar: -el $n$ utilizado, aunque algunos expertos postulan que éste debe corresponder a lo menos al doble de ítemes (23), lo cual se cumple en esta investigación. Sumado a que las pruebas para evaluar la validez de los análisis utilizados resultaron significativas con los datos obtenidos. -El grupo de estudio estuvo constituido por personas de diversas ocupaciones del área de la salud, todos/as se desempeñan en el ámbito académico, lo que podría originar el supuesto de menor variabilidad de los datos. -Finalmente se destaca que ESCAM es un instrumento relativamente nuevo, el cual está en fase de desarrollo (6), por lo que no se disponen de suficientes criterios empíricos con que comparar los resultados. Por estas razones, es necesario realizar nuevas investigaciones con esta escala con el fin de disminuir estas limitaciones.

ESCAM es un instrumento fiable y válido para evaluar el constructo Carga Mental de
Trabajo (CMT). Los análisis estadísticos realizados apoyan la idea de que la CMT es un constructo multidimensional. Se indica que la solución factorial resultante con los datos obtenidos no excluyen la existencia de otras soluciones en otro tipo de muestras, por lo que el mejor ajuste debe ser evaluado a través de nuevos análisis factoriales a futuro.

\section{REFERENCIAS}

1. Ferrer R, Dalmau I. Revisión del concepto de carga mental: evaluación, consecuencia y proceso de normalización. Anuario de psicología. 2004; 35(4): 521-45.

2. Ramírez C. Ergonomía y productividad. 2a ed. México: LIMUSA; 2006.

3. Rubio S, Luceño L, Martín J, Jaén M. Modelos y procedimientos de evaluación de la carga mental de trabajo. EduPsykhé. 2007; 6(1): 85-108.

4. Gil-Monte P. Riesgos psicosociales en el trabajo y salud ocupacional. Rev Perú Med Exp Salud Pública. 2012; 29(2): 23741.

5. Díaz C. Actividad laboral y carga mental de trabajo. Ciencia y trabajo. 2010; 12(36): 281-92.

6. Rolo G, Díaz D, Hernández E. Desarrollo de una escala subjetiva de carga mental de trabajo (ESCAM). Rev psicol trab organ. 2009; 25(1): 29-37.

7. O’Donnell C, Eggemeier F. Workload assessment methodology. En: Boff K, Kaufman L, Thomas J; editores. Handbook of perception and human performance. Vol. II, Cognitive Processes and Performance. New York: John Wiley \& Sons Inc; 1986. 1296 p.

8. Rubio S, Díaz E, Martín J. Aspectos metodológicos de la evaluación subjetiva de la carga mental de trabajo. Arch Prev Riesgos Labor. 2001; 4(4): 160-68.

9. Cañas J, Waerns Y. Ergonomía cognitiva. Aspectos psicológicos de la interacción 
de las personas con la tecnología de la información. Madrid, España: Ed. Médica Panamericana. 2001. 260 p.

10. Neill D. Nursing workload and the changing health care environment: a review of the literature. Administrative Issues Journal. 2011; 1(2): 132-43.

11. Hacker W. Carga mental de Trabajo. En: Enciclopedia de salud y seguridad en el trabajo, Organización Internacional del Trabajo (OIT) [Internet]. Madrid: Instituto Nacional de Seguridad e Higiene en el Trabajo; 2001 [citado 17 de agosto 2014]. Disponible en: http://www.insht. es

12. Cañas J. Personas y Máquinas. El diseño de su interacción desde la ergonomía cognitiva. Madrid: Ed. Pirámide; 2004. p. 105-20.

13. Díaz D, Hernández E, Rolo G, Galván E, Fraile, M, Loayssa G. Escala Subjetiva de Carga Mental (ESCAM) [Internet]. Tenerife: Instituto Canario de Seguridad Laboral; 2010 [citado 17 agosto 2014]. Disponible en: http://www.gobiernodecanarias.org/cicnt/icasel/documentos/ manualcarga_mental.pdf

14. Sánchez R, Etcheverry J. Validación de escalas de medición en salud. Rev Salud Pública. 2004; 6(3): 302-18.

15. Polit D, Hungler B. Investigación científica en ciencias de la salud. 6a ed. México DF: McGraw-Hill / Interamericana de México; 2000. 725 p.

16. Hernández R, Fernández C, Baptista $P$.
Metodología de la investigación. 5a ed. México DF: McGraw-Hill: 2010. 656 p.

17. Oviedo H, Campos A. Aproximación al uso del coeficiente alfa de Cronbach. Rev Colomb Psiquiatr. 2005; 34(4): 572-80.

18. Landero R, González M (editores). Estadística con SPSS y metodología de la investigación. México: Trillas; 2006.

19. Saavedra N, Fuentealba C, Pérez J. Cuestionario de evaluación de riesgos psicosociales en el trabajo SUSESO-ISTAS 21. Santiago, Chile: Unidad de comunicación y extensión SUSESO, Superintendencia de Seguridad Social, Gobierno de Chile; 2009.

20. Montoya O. Aplicación del análisis factorial a la investigación de mercados, casos de estudio. Scientia et técnica. 2007; 13(35): 281-86.

21. Vilaret A, Ortiz A. Carga Mental y Síndrome de Burnout en docentes a tiempo completo de una universidad de Quito. Revista EÍDOS. 2013; 6: 36-47.

22. Rusell D. In search of underlying dimensions: the use (and abuse) of factor análisis in personality and social psychology bulletin. Pers Soc Psychol Bull. 2002; 28(12): 1629-46.

23. Morales P. El análisis factorial en la construcción e interpretación de test, escalas y cuestionarios [Internet] Madrid: Universidad Pontificia Comillas; 2011 [citado 17 agosto 2014]. Disponible en: http://www. upcomillas.es/personal/peter/investigacion/AnalisisFactorial.pdf 